Original Study

\title{
Effects of the FIT-HIP Intervention for Fear of Falling After Hip Fracture: A Cluster-Randomized Controlled Trial in Geriatric Rehabilitation
}

\author{
Maaike N. Scheffers-Barnhoorn $\mathrm{MD}^{\mathrm{a}}{ }^{\text {,* }}$, Monica van Eijk MD, PhD ${ }^{\mathrm{a}}$, \\ Jolanda C.M. van Haastregt PhD ${ }^{\mathrm{b}}$, Jos M.G.A. Schols MD, PhD ${ }^{\mathrm{b}, \mathrm{c}}$, \\ Romke van Balen MD, $\mathrm{PhD}^{\mathrm{a}}$, Nan van Geloven $\mathrm{PhD}^{\mathrm{d}}$, \\ Gertrudis I.J.M. Kempen $\mathrm{PhD}^{\mathrm{b}}$, Wilco P. Achterberg MD, $\mathrm{PhD}^{\mathrm{a}}$

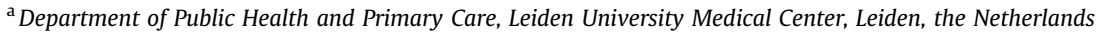 \\ ${ }^{\mathrm{b}}$ Department of Health Services Research and Care and Public Health Research Institute (CAPHRI), Maastricht University, Maastricht, the Netherlands \\ ${ }^{\mathrm{c}}$ Department of Family Medicine and Care and Public Health Research Institute (CAPHRI), Maastricht University, Maastricht, the Netherlands \\ ${ }^{\mathrm{d}}$ Department of Medical Statistics and Bioinformatics, Leiden University Medical Center, Leiden, the Netherlands
}

\section{Keywords:}

Fear of falling

hip fracture

geriatric rehabilitation

cognitive behavioral therapy

randomized controlled trial

\begin{abstract}
A B S T R A C T
Objectives: Fear of falling (FoF) is common after hip fracture and can impede functional recovery because of activity restriction. The Fear of falling InTervention in HIP fracture geriatric rehabilitation (FIT-HIP intervention) was designed to target FoF and consequently to improve mobility. The aim of this study was to evaluate the effect of the FIT-HIP intervention in patients with FoF in geriatric rehabilitation (GR) after hip fracture.

Design, setting, and participants: This cluster-randomized controlled trial was performed in 11 post-acute GR units in the Netherlands (2016-2017). Six clusters were assigned to the intervention group, 5 to the usual care group. We included 78 patients with hip fracture and FoF (aged $\geq 65$ years; 39 per group). Intervention(s): The FIT-HIP intervention is a multicomponent cognitive behavioral intervention conducted by physiotherapists, embedded in usual care in GR. The FIT-HIP intervention was compared to usual care in GR.

Measurements: FoF was assessed with the Falls Efficacy Scale-International (FES-I) and mobility, with the Performance Oriented Mobility Assessment (POMA). Data were collected at baseline, discharge, and 3 and 6 months postdischarge from GR. Primary endpoints were change scores at discharge. Linear mixed models were used to evaluate the treatment effect.

Results: No significant between-group differences were observed for primary outcome measures. With the usual care group as reference, the FES-I estimated difference between mean change scores was 3.3 [95\% confidence interval $(\mathrm{CI})-1.0,7.5, P=.13$ ] at discharge from $\mathrm{GR} ;-4.1(95 \% \mathrm{CI}-11.8,3.6, P=.29)$ after 3 months; and $-2.8(95 \% \mathrm{CI}-10.0,4.4, P=.44)$ after 6 months. POMA estimated difference was -0.3 (95\% CI $-6.5,5.8, P=.90)$.

Conclusion/Implications: The FIT-HIP intervention was not effective in reducing FoF. Possibly FoF (shortly) after hip fracture can to some extent be appropriate. This may imply the study was not able to accurately identify and accordingly treat FoF that is maladaptive (reflective of disproportionate anxiety).
\end{abstract}

(C) 2019 AMDA - The Society for Post-Acute and Long-Term Care Medicine.
This work was supported by ZonMw (The Netherlands Organization for Health Research and Development; research grant number 839120004). This study has been conducted independently of the funding body. SBOH (employer of elderly care medicine trainees) and the Leiden University Medical Center (training center for older elderly medicine) additionally support this study.

The authors declare no conflicts of interest.
* Address correspondence to Maaike N. Scheffers-Barnhoorn, MD, Department of Public Health and Primary Care, Leiden University Medical Center, Postbox 9600, Leiden 2300 RC, the Netherlands.

E-mail address: m.n.scheffers-barnhoorn@lumc.nl (M.N. Scheffers-Barnhoorn). 
Despite advances made in both the acute care for hip fracture patients and post-acute rehabilitation services provided, ${ }^{1-6}$ long-term functional recovery after hip fracture still remains limited., ${ }^{7,8}$ Although many factors can impede recovery after hip fracture, only a few are potentially modifiable. ${ }^{9-12}$ In this regard, fear of falling (FoF), defined as "a lasting concern about falling that leads to an individual avoiding activities that he/she remains capable of performing," may be an important risk factor. ${ }^{13}$ More than $50 \%$ of patients who have sustained a hip fracture express FoF. ${ }^{14-16}$ FoF can hamper progress in functional performance as a result of avoidance of activities. ${ }^{14}$ Moreover, it is associated with an increased risk of falling, decreased mobility, loss of independence, institutionalization, and lower quality of life and social participation. ${ }^{14,17,18}$ Therefore, treatment of FoF after hip fracture may be a key element in approaches to improve functional recovery after fracture.

In the past decades, several interventions for community-dwelling older adults with FoF have been developed and evaluated. ${ }^{19,20}$ These programs often use a cognitive behavioral approach, including cognitive restructuring, personal action plans to encourage engagement and physical activity, exposure in vivo (eg, practicing activities in fear-related real-life situations), information on fall prevention, and motivational interviewing. One of these programs is the widely used intervention program "A Matter of Balance," which has proven to be (cost-) effective to reduce FoF in community-dwelling older adults in the United States and the Netherlands. ${ }^{21-26}$ However, none of the programs available focus on patients with hip fracture.

In the Netherlands, specialized inpatient multidisciplinary rehabilitation services for frail older patients are organized as "geriatric rehabilitation" (GR) within post-acute GR units. ${ }^{27}$ The rehabilitation program includes physical and occupational therapy, and treatment of comorbidities. Approximately half of all older adults with hip fracture in the Netherlands are referred to GR following surgical repair of the fracture. In order to adequately address FoF in this specific population, it is essential that intervention strategies are designed to fit the rehabilitation setting. For this purpose, the cognitive behavioral approach used in the Dutch version of "A Matter of Balance" was adapted to an individualized tailor-made intervention: the Fear of falling InTervention in HIP fracture geriatric rehabilitation (ie, the FITHIP intervention). ${ }^{28}$ The FIT-HIP intervention is incorporated in the multidisciplinary GR treatment program.

This study aims to evaluate whether the FIT-HIP intervention is effective in reducing FoF and, consequently, improving mobility when compared to care as usual in GR.

\section{Methods}

This cluster-randomized controlled trial (RCT) compared usual care in inpatient multidisciplinary GR in the Netherlands to usual care combined with the FIT-HIP intervention. ${ }^{28}$ Eleven GR units (clusters) were recruited to participate. The Ethics Committee of the Leiden University Medical Center (LUMC) approved the study protocol (P15.212; 09-09-2015), which is registered in the Netherlands Trial Register (No. XXX).

\section{Recruitment of Participants}

All patients admitted to the participating GR units due to recent hip fracture were screened for eligibility in the first week of admission. Inclusion criteria were age $\geq 65$ years and at least sometimes being concerned to fall, based on a 1-item question, namely, "Are you concerned to fall?" (answer options: never, almost never, sometimes, often, very often).

Exclusion criteria were (1) the presence of a condition interfering with learnability [formal diagnosis of dementia; major psychiatric disease or a score $>1$ on the Hetero-anamnesis List Cognition (HAC), ${ }^{29}$ which is suggestive for premorbid cognitive disabilities]; (2) prefracture Barthel index score $<15$; (3) pathologic hip fracture; (4) life expectancy $<3$ months; and (5) insufficient mastery of the Dutch language.

All participants provided written informed consent to participate prior to baseline assessment.

\section{Randomization and Blinding}

Following recruitment of all participating GR units, 5 were randomly assigned to the usual care group and 6 to the intervention group. Computer-generated randomization was performed by an independent researcher of the LUMC using the random generator of SPSS (version 23.0).

Outcome measures were assessed by independent research assistants blinded to group allocation. Health care professionals were instructed not to inform the participants about allocation status. In a further attempt to conceal treatment allocation for participants, at enrollment, all participants received a 4-page information brochure on FoF. ${ }^{28}$ As health care strategies directed at reducing risk of falling in older adults based on educational interventions alone have not proven effective, ${ }^{30}$ we expected this to serve as a suitable dummy intervention.

\section{Interventions}

\section{FIT-HIP Intervention}

Details of the intervention have been published previously ${ }^{28}$; a summary is presented in Table 1. The FIT-HIP intervention consists of various cognitive behavioral elements aimed at reducing the FoF, including psycho-education, guided exposure to feared activities, cognitive restructuring, and relapse prevention. The intervention is integrated in the physical therapy sessions and combined with the regular exercise training in GR.

From each GR unit allocated to the intervention group, 2 physiotherapists were trained to conduct the intervention. All intervention units additionally provided a psychologist to counsel the physiotherapists as needed, specifically with regard to the "cognitive restructuring." During monthly meetings organized by each GR team individually, the physiotherapists and psychologists discussed the participants' progress and the challenges in the treatment. Physiotherapists were also encouraged to directly consult their "buddy" psychologist if they encountered difficulties during treatment. Furthermore, the nursing staff was briefed on the background and rationale of guided exposure to help them incorporate these principles in their work and to adhere to the "FIT-HIP fear ladders" (Table 1).

\section{Usual Care}

In the Netherlands, inpatient geriatric rehabilitation is multidisciplinary care led by an elderly care physician. ${ }^{31}$ General aspects of physiotherapy treatment include training of mobility, balance and gait, and exercise to improve muscle strength. Nursing staff and an occupational therapist are involved to help improve self-care by coaching patients to perform basic activities of daily living (ADL), such as transferring and bathing. ${ }^{15,27}$ In general, a patient receives 5 to 6 physiotherapy sessions per week, although therapy intensity may vary because of variations in patients' physical endurance and the formalized agreements on therapy intensity employed by the GR units.

\section{Outcome Measures}

Baseline (T0) and discharge (T1) assessments were performed in the first and last week of the GR trajectory by means of structured face-to-face interviews and task-oriented physical tests. Follow-up 
Table 1

Overview of the FIT-HIP Intervention

\begin{tabular}{|c|c|}
\hline Element & Description \\
\hline Guided exposure-rationale & $\begin{array}{l}\text { Guided exposure is the graded and repeated exposure to situations that give rise to fear (of falling). As recurrent } \\
\text { exposure to the feared situation or activity is performed under supervision and in a manner that is predictable } \\
\text { and controllable, this leads to the positive experience that the fear gradually fades out as the activity is } \\
\text { practiced more often. After the fear for this specific situation has subsided, the exposure can be extended to the } \\
\text { "next level," practicing the activity in a manner that leads to a greater level of fear (fear hierarchy for graded } \\
\text { exposure). For fear of falling (FoF), the feared activities will be situations concerning physical activity. In the } \\
\text { rehabilitation after hip fracture, this will predominantly be basic activities in daily living, such as transferring, } \\
\text { standing, and walking. }\end{array}$ \\
\hline
\end{tabular}

Implementation in the FIT-HIP intervention

In the FIT-HIP intervention, the physiotherapist helps the participant assess situations that give rise to FoF [within the first week of admission to geriatric rehabilitation (GR)]. For each "feared" activity the physiotherapist and participant draft a fear hierarchy, designed as a "fear ladder" (template example published in protocol). ${ }^{28}$ The FIT-HIP fear ladder consists of 6 "steps," each step representing a functional goal. The functional goal describes in which manner the activity is practiced or performed. The goals are ranked with an increasing level of FoF as the activity gets more complex (or has to be performed with less assistance). The FITHIP fear ladders are the guiding principle for the multidisciplinary approach to apply guided exposure for all aspects of mobilization. The physiotherapist evaluates the fear ladders with the participant weekly, and the fear ladders are revised on the basis of progress (reduction of FoF).

Intervention provider(s)*

Physiotherapists during physical therapy sessions; as applicable, by nursing staff when assisting patients in basic activities of daily living that give rise to FoF; nursing staff assisting participants in practicing "fearful" activities as "homework assignments" after physical therapy.

Schedule

Cognitive restructuring - rationale

ncorporated in all physical therapy sessions (and nursing care activities) for the duration of inpatient multidisciplinary GR as long as FoF persists.

Thoughts (and associated beliefs) influence how a person feels and accordingly how a person appraises and responds to a situation. Excessive concern to fall (fear of falling) can be based on unrealistic thoughts and beliefs with regard to (risk of) falling. This excessive FoF may lead to avoidance of (physical) activity and consequently fortify the FoF. Cognitive restructuring is a technique used to explore thoughts and beliefs and therefore to identify, challenge, and modify unrealistic thoughts. In the FIT-HIP intervention, participants are coached to explore their thoughts concerning physical activity and fall risk. In doing so, they are encouraged to identify maladaptive and unrealistic thoughts and in turn formulate and apply more realistic thoughts. The principle of (un)realistic thoughts is also incorporated into the relapse prevention plan (see below).

Implementation in the FIT-HIP intervention

Physiotherapists are trained to guide the participant in exploring their thoughts concerning physical activity and (risk of) falling. A worksheet is used to structure the process of cognitive restructuring and to provide the participant insight in this process (analyzing the situation and the associated thoughts, feelings, behavior, and consequences and subsequently formulating more realistic thoughts).

Intervention provider(s)

Physiotherapists; a psychologist is trained as a "buddy" to coach the physiotherapists in these principles as and when additional help is needed.

Schedule

During at least 1 physical therapy session, the cognitive restructuring is applied and practiced with the participant. Subsequently, the participant is encouraged to fill in the worksheet as a "homework assignment." This is reviewed and discussed during the next therapy session. These "key" thoughts can briefly be recapitulated in situations when the FoF is noticeable in the physical therapy sessions. The process of cognitive restructuring can be repeated as needed (when the FoF persists).

Psycho-education-rationale and implementation in the FIT-HIP intervention

The psycho-education is used to reinforce the various elements of the FIT-HIP intervention. In the initial phase of GR, the participant receives information on anxiety, (consequences and treatment of) FoF, and the rationale and background of guided exposure and cognitive restructuring. In the final phase of GR, when discharge home is being planned, the psycho-education focuses on home safety. The information on home safety is also processed in the relapse prevention plan (see below).

Physiotherapists discuss the information with the participant.

During at least 2 physical therapy sessions (one in the initial phase of rehabilitation; the other preceding the discharge home); as applicable, the psycho-education can additionally be incorporated in the therapy sessions, related to situations occurring during therapy (eg, fall prevention).

The relapse prevention is aimed at helping the participant to anticipate and cope with relapse to FoF.

In the FIT-HIP intervention, the relapse prevention is designed to optimize the transition to predominantly independent living circumstances after discharge home. For this purpose, a "relapse prevention plan" is composed together with the participant. This "Staying Active Plan" aims at preparing the participant for challenging situations in which there is a risk for relapse to FoF and activity restriction. The Staying Active Plan consists of (information on) (1) general home safety and fall prevention; (2) individualized advice for safe ambulation and how to stay active; (3) preventing, recognizing, and dealing with a relapse [including notice of ([mal]adaptive) thoughts]. The information is discussed together with the participant and presented in writing as a reference book.

In addition, a telephonic booster is conducted 6 weeks after discharge from GR. The telephonic booster is aimed at evaluating the FoF (and activity restriction). If necessary, advice is given how to deal with FoF, in addition to the prior advice formulated in the Staying Active Plan.

Both the Staying Active Plan and telephonic booster are conducted by physiotherapists.

Intervention provider(s)

Schedule

During at least 1 physical therapy session during GR (Staying Active Plan) and 1 telephonic booster session after discharge home.

Physiotherapists are trained* in motivational interviewing techniques to assist the participant in the process of behavior change. These techniques help the physiotherapist gain insight into the participant's extrinsic and intrinsic motivation and explore which rehabilitation goals are important for the participant, in order to personalize treatment goals in the FIT-HIP intervention.

\footnotetext{
*Physiotherapists received 2 training sessions (4 hours each), and psychologists, one 4-h session (together with physiotherapists).
} 
assessments at 3 and 6 months after discharge (T2/T3) were performed by postal questionnaires. Missing data at follow-up and the Functional Ambulation Categories (see below) were acquired by telephonic interviews.

The purpose of the FIT-HIP intervention is to reduce FoF in order to improve physical functioning. Therefore, the FIT-HIP trial had 2 primary outcome measures: (1) the post-intervention change in FoF measured with the Falls Efficacy Scale-International (FES-I), ${ }^{32}$ and (2) change in mobility function assessed with the Performance Oriented Mobility Assessment (POMA). ${ }^{33}$

The FES-I is a 16-item instrument, scored on a 4-point Likert scale, assessing FoF (defined as concerns about falling) related to basic and more demanding physical and social activities. The total score on the FES-I ranges from 16 to 64 , with higher scores indicating a higher level of FoF. The FES-I has good reliability and validity, and its use has been validated in Dutch patients with hip fracture in GR. ${ }^{32}$

The POMA is a reliable and valid clinical examination tool that assesses gait and balance ability as a measure for mobility in older adults. $^{33}$ It consists of a 9-item balance scale and a 7-item gait scale. The total score ranges from 0 to 28 , with lower scores indicating a greater risk of falling. In the event the participant was unable to perform the test because of physical impairment, we set the score to 0 . The POMA was assessed at baseline and at discharge, whereas the FES-I was assessed at all 4 measurement points.

Secondary outcome measures were self-reported activity restriction due to FoF and (in)dependence in walking ability measured by the Functional Ambulation Categories (FAC). Activity restriction due to FoF was assessed with a 1-item question, "Do you avoid activities due to fear of falling?" Response categories were never (0), almost never (1), sometimes (2), often (3), and very often (4). The FAC evaluates ambulation ability on a 6-point ordinal scale, describing the degree of support needed when walking; this scale ranges from nonfunctional walking (score 0 ) to independent walking on all surfaces (score 5).

\section{Additional Variables}

At baseline, we collected sociodemographic data and information on various aspects of physical and mental health for descriptive purposes (Table 2). To compare the therapy intensity in both groups, we collected data at participant level on frequency and duration of all therapy provided in GR (information obtained from routine data registration used for reimbursement purposes). Adverse events such

Table 2

Baseline Characteristics of the FIT-HIP Participants and Characteristics of Geriatric Rehabilitation Outcomes

\begin{tabular}{|c|c|c|c|c|}
\hline & All Participants $(\mathrm{n}=77)$ & Usual Care Group ( $\mathrm{n}=38$ ) & Intervention Group $(\mathrm{n}=39)$ & $P$ Value* \\
\hline \multicolumn{5}{|l|}{ Demographic data } \\
\hline Age, y, mean (SD) & $82.5(7.6)$ & $81.3(7.9)$ & $83.7(7.3)$ & .18 \\
\hline Female gender, n (\%) & $61(79.2)$ & $27(71.1)$ & $34(87.2)$ & .98 \\
\hline Living alone, $\mathrm{n}(\%)$ & $51(66.2)$ & $24(63.2)$ & $27(69.2)$ & .64 \\
\hline \multicolumn{5}{|c|}{ Comorbidity, functional status, and physical functioning } \\
\hline $\mathrm{FCI}(0-18),{ }^{\dagger} \operatorname{median}(\mathrm{IQR})^{\ddagger}$ & $3.0(1.0-5.0)$ & $2.5(1.0-4.0)$ & $3.5(1.8-6.0)$ & .05 \\
\hline 10MWT, m/s, median (IQR) & $0.0(0.0-0.3)$ & $0.0(0.0-0.5)$ & $0.0(0.0-0.3)$ & .27 \\
\hline Prefracture activity restriction due to fear of fa & & & & .72 \\
\hline Never/almost never/sometimes & $70(90.9)$ & $36(94.7)$ & $34(87.2)$ & \\
\hline Often/very often & $7(9.1)$ & $2(5.3)$ & $5(12.8)$ & \\
\hline \multicolumn{5}{|l|}{ (Neuro)psychological factors, median (IQR) } \\
\hline GDS- 8 total score $(0-8)^{\dagger}$ & $0.0(0.0-2.0)$ & $0.0(0.0-2.0)$ & $1.0(0.0-2.0)$ & .93 \\
\hline HADS-A total score $(0-21)^{\dagger}$ & $3.0(1.0-6.8)$ & $3.0(1.0-5.3)$ & $4.0(1.0-8.3)$ & .36 \\
\hline MMSE $(0-30)^{\S}$ & $27.0(25.0-29.0)$ & $27.0(25.0-29.0)$ & $27.0(24.0-29.0)$ & .74 \\
\hline \multicolumn{5}{|l|}{ Primary outcomes } \\
\hline \multicolumn{5}{|l|}{ FES-I (0-64) ${ }^{\dagger}$ mean (SD) } \\
\hline Baseline: T0 & $34.2(10.6)$ & $34.4(11.4)$ & $33.9(9.9)$ & \multirow[t]{4}{*}{.84} \\
\hline Discharge: $\mathrm{T} 1$ & $29.9(10.0)$ & $27.0(8.2)$ & $32.8(11.0)$ & \\
\hline 3-month follow-up: T2 & $35.9(13.0)$ & $36.6(12.4)$ & $35.1(13.9)$ & \\
\hline 6-month follow-up: T3 & 36.5 (11.9) & 36.5 (11.9) & $36.5(12.1)$ & \\
\hline \multicolumn{5}{|l|}{ POMA (0-28), median (IQR) } \\
\hline POMA baseline: T0 & $0.0(0.0-8.0)$ & $0.0(0.0-10.5)$ & $0.0(0.0-4.0)$ & \multirow[t]{2}{*}{.13} \\
\hline POMA discharge: $\mathrm{T} 1^{\ddagger}$ & $17.0(13.0-20.0)$ & $18.0(13.8-21.0)$ & $17.0(12.5-20.0)$ & \\
\hline \multicolumn{5}{|c|}{ Secondary outcomes } \\
\hline \multicolumn{5}{|c|}{ Activity restriction due to fear of falling $(0-4),{ }^{\dagger}$ median (IQR) } \\
\hline Baseline: T0 & $0.0(0.0-2.0)$ & $0.0(0.0-1.3)$ & $0.0(0.0-2.0)$ & \multirow[t]{4}{*}{.43} \\
\hline Discharge: $\mathrm{T} 1$ & $0.0(0.0-1.0)$ & $0.0(0.0-0.3)$ & $0.0(0.0-1.0)$ & \\
\hline 3-month follow-up: T2 & $2.0(1.0-3.0)$ & $2.0(1.0-2.5)$ & $2.0(1.0-3.0)$ & \\
\hline 6-month follow-up: T3 & $2.0(1.0-2.3)$ & $2.0(1.0-3.0)$ & $2.0(0.3-2.0)$ & \\
\hline \multicolumn{5}{|l|}{ FAC $(0-5),{ }^{\S}$ median $(\mathrm{IQR})$} \\
\hline Baseline: T0 & $2.0(1.0-3.0)$ & $3.0(2.0-4.0)$ & $2.0(0.0-3.0)$ & \multirow[t]{4}{*}{.002} \\
\hline Discharge: $\mathrm{T} 1$ & $4.0(4.0-5.0)$ & $4.0(4.0-5.0)$ & $4.0(4.0-4.3)$ & \\
\hline 3-month follow-up: T2 & $5.0(4.0-5.0)$ & $5.0(4.0-5.0)$ & $4.5(4.0-5.0)$ & \\
\hline 6-month follow-up: T3 & $5.0(4.0-5.0)$ & $5.0(4.0-5.0)$ & $5.0(4.0-5.0)$ & \\
\hline \multicolumn{5}{|l|}{ GR outcome characteristics } \\
\hline Discharge home after GR, n (\%) & $63(94.0)$ & $32(97.0)$ & $31(91.2)$ & 61 \\
\hline Duration of admission to $\mathrm{GR}, \mathrm{d}$, median (IQR) & $38.0(31.0-64.0)$ & $37.0(21.0-63.0)$ & $42.5(34.0-64.5)$ & .09 \\
\hline
\end{tabular}

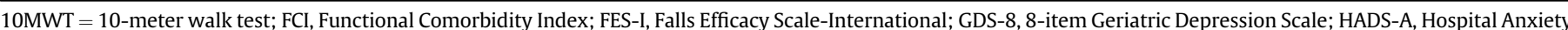

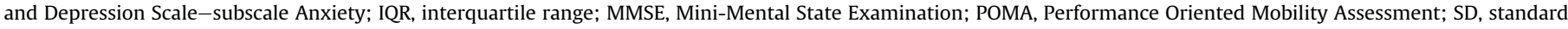
deviation.

Self-reported activity restriction due to fear of falling; scores indicate never (0), almost never (1), sometimes (2), often (3) and very often (4).

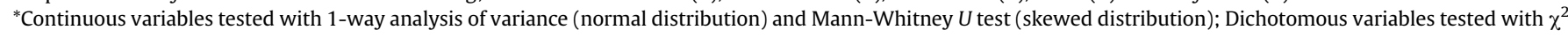
test.

'Lower scores indicate better status.

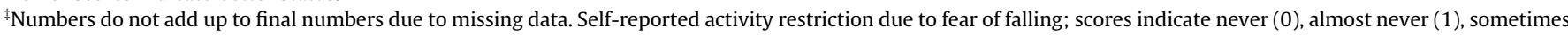
(2), often (3) and very often (4).

${ }^{8}$ Higher scores indicate better status. 
as fall incidents, hospital readmissions, and death were registered by attending elderly care physicians during GR. During follow-up, this information was assessed with the questionnaires sent to participants.

\section{Sample Size}

As the ultimate goal to treat FoF is to improve functional recovery after hip fracture, we chose to use POMA to calculate sample size. To have $80 \%$ power to detect a statistically significant and minimal clinically relevant difference of 3.8 in the POMA score between groups at discharge from GR, we needed 40 participants per group (corresponding means 17.0 and 20.8, respectively; standard deviation per group 6.0); this was based on an alpha of $5 \%$ (2-sided). The intraclass correlation was set to 0.05 to account for the effect of cluster randomization. To additionally account for a minimum of $10 \%$ loss to follow-up at discharge, we planned to recruit 15 participants per cluster.

\section{Statistical Analysis}

All analyses were performed on an intention-to-treat basis using IBM SPSS Statistics for Windows version 23.0. Statistical significance was set at $P<.05$ (2-sided). The mean change score in outcome measure from baseline (score $\mathrm{Tx}$-score $\mathrm{T} 0$ ) was used for all effectiveness analyses. These within-group differences were compared between the treatment groups using a linear mixed model to account for the clustering of participants within the GR units. In this model, we also corrected for the imbalance found between treatment groups with regard to the baseline FAC score and Functional Comorbidity Index (FCI). Both delayed postoperative ambulation and comorbidity are established risk factors for negative outcomes after hip fracture. ${ }^{9,10,34}$ The treatment effect is presented as an estimated difference between the mean change score per group (with corresponding 95\% confidence interval), with the usual care group as reference.

In addition to this primary, most extensive linear mixed model, we evaluated treatment effects solely adjusted for (1) baseline value of outcome measure; (2) baseline value of outcome measure + baseline FAC score; and (3) baseline value of outcome measure and baseline FCI score. Detailed information on the linear mixed models is presented in Appendix Table A1.

\section{Results}

Figure 1 presents the participant flow chart of the FIT-HIP trial. Participants were recruited between March 2016 and January 2017. Because of a limited inclusion rate (despite extending the recruitment period by 2 months), only 78 participants were included (39 in each group). Both groups had a similar drop-out rate during the study-frequently related to health problems. No GR units withdrew participation during the trial.

Table 2 presents the baseline characteristics of the study population; mean age was 83 (standard deviation $=7.6$ ) years, and the majority of the participants were female (79\%) and lived alone prior to fracture (66\%). The treatment groups differed in baseline ambulation function and comorbidity count (median FAC score: 3, and 2, respectively, in the usual care group and the intervention group, $P=.002$; median FCI score: 2.5 and 3.5, respectively, in the usual care group and the intervention group, $P=.05$ ). Both groups had a similar length of stay in GR and similar rates of discharge home. No significant differences were identified with regard to therapy intensity (total amount of treatment in GR; physiotherapy and treatment by psychologist; data not shown).

Table 3 presents the results of the primary models, adjusted for baseline score of outcome measure, baseline FAC, and FCI. Data from the less extensive models are presented in Appendix Table A2, and only differed from the primary model with regard to the FES-I score at $\mathrm{T} 1$.

\section{Primary Outcome Measures: Fear of Falling and Mobility}

At discharge from GR, the usual care group showed a decrease in FoF compared to the intervention group. However, this difference was not significant (estimated difference between the mean change scores for FES-I score of 3.3, 95\% confidence interval $-4.1,3.6, P=.13$ ), and did not persist after discharge. In the usual care group, the FES-I score increased to a greater extent, leading to comparable levels of FoF in both groups during follow-up (Table 3 and Figure 2). At discharge, no differences between groups were found for the POMA change score (estimated difference between mean change scores $-0.3,95 \%$ confidence interval $-6.5,5.8, P=.90)$.

\section{Secondary Outcomes}

Both groups had a slight decrease in activity restriction due to FoF at discharge, and a subsequent increase during follow-up. The outcome scores were comparable. The 2 groups did not differ with regard to ambulation function (Table 3 ).

\section{Harms or Adverse Events}

Comparable rates of mortality and hospital readmissions were found in both groups (Appendix Table A3). However, more fall events and participants encountering $>1$ fall event were identified in the usual care group.

\section{Discussion}

This cluster RCT among patients with hip fracture found no positive effects of the FIT-HIP intervention on the primary outcome measures for mobility (POMA) and fear of falling (FES-I) when compared to usual care. At discharge from GR, the usual care group had a greater reduction in FES-I score compared with the intervention group. However, this difference did not persist over time and, because of a trend toward a greater increase of FoF in the usual care group, this resulted in comparable levels for both groups at 3 and 6 months after discharge. Additionally, no differences were observed between the groups with regard to ambulation (FAC) and self-reported activity restriction due to FoF.

Inappropriate timing of screening and treatment of FoF is perhaps the most important explanation for the finding that the FIT-HIP intervention did not prove to be beneficial in reducing FoF. Although the negative effect of FoF on physical and functional outcome after hip fracture is well established, ${ }^{14,35-37}$ a recent study provided insight into the course of FoF after hip fracture in relation to long-term physical functioning. ${ }^{16}$ These latter results confirm findings from Oude Voshaar and colleagues and illustrate that FoF that presents shortly after the fracture (2-4 weeks) is not predictive for poorer long-term physical performance (6-12 months), which is in contrast to FoF that is present 6 to 12 weeks after the fracture. ${ }^{13,16}$ This suggests a time-mediated effect for FoF after fracture. Accordingly, this may indicate that FoF that occurs in the initial course of rehabilitation after hip fracture can be transient and may even represent an adaptive and normal response to the sudden impairment in physical condition (including reduced balance function), as opposed to FoF that persists or arises at a later stage. In the present trial, we assessed FoF in the first week of GR. Generally, this represents the second week after fracture (because of an average hospital admission of 1 week). Treatment started directly after enrollment and was administered only during inpatient GR (with the exception of the telephonic booster postdischarge).$^{28}$ An inpatient 


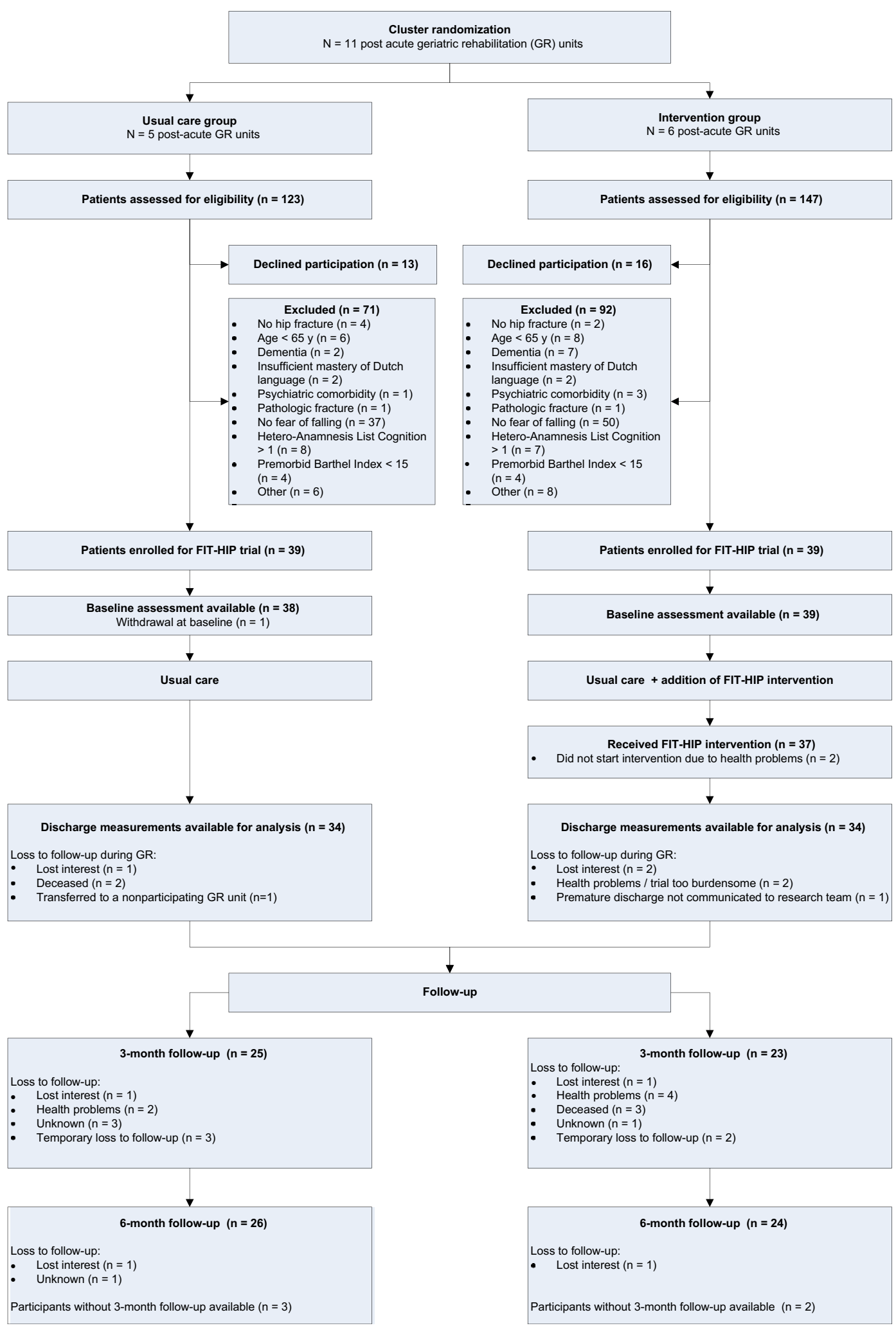

Fig. 1. Flow diagram of the FIT-HIP study.

GR program for hip fracture patients usually lasts 6 to 7 weeks, ${ }^{15,27}$ as was also the case in our study. This implies that the FIT-HIP intervention mainly targets the initial phase of recovery after fracture in which FoF does not seem to be associated with negative long-term effects on functional outcome.
If we then postulate that FoF does not by definition solely have negative effects, and may under certain circumstances be an adaptive response, it may be of interest to (re)consider the role of anxiety in the context of fall-related concerns. Anxiety has been associated with (higher levels of) FoF, both in community-dwelling older adults ${ }^{38}$ and 
Table 3

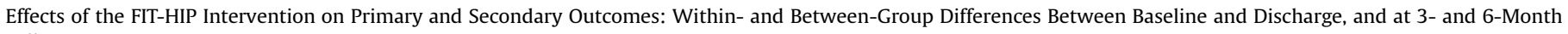
Follow-up

\begin{tabular}{|c|c|c|c|c|c|c|c|c|c|c|c|c|c|c|}
\hline & \multirow{2}{*}{\multicolumn{2}{|c|}{$\frac{\text { Baseline Value }}{\text { T0 }}$}} & \multicolumn{6}{|c|}{ Within-Group Differences } & \multicolumn{6}{|c|}{ Between-Group Differences: Intervention vs Usual Care Group* } \\
\hline & & & \multicolumn{2}{|c|}{$\Delta \mathrm{T} 1-\mathrm{T} 0$} & \multicolumn{2}{|c|}{$\Delta \mathrm{T} 2-\mathrm{T} 0$} & \multicolumn{2}{|c|}{$\Delta \mathrm{T} 3-\mathrm{T} 0$} & \multicolumn{2}{|l|}{$\mathrm{T} 1$} & \multicolumn{2}{|l|}{$\mathrm{T} 2$} & \multicolumn{2}{|l|}{ T3 } \\
\hline & $\mathrm{n}$ & Mean (SD) & $\mathrm{n}$ & Mean (SD) & $\mathrm{n}$ & Mean (SD) & $\mathrm{n}$ & Mean (SD) & $\begin{array}{l}\text { Adj Mean Diff } \\
(95 \% \mathrm{CI})\end{array}$ & $P$ Value & $\begin{array}{l}\text { Adj Mean Diff } \\
(95 \% \mathrm{CI})\end{array}$ & $P$ Value & $\begin{array}{l}\text { Adj Mean Diff } \\
(95 \% \mathrm{CI})\end{array}$ & $P$ Value \\
\hline \multicolumn{15}{|l|}{ Primary outcomes } \\
\hline \multicolumn{15}{|l|}{ FES-I (16-64) } \\
\hline Usual care & 37 & $34.4(11.4)$ & 33 & $-6.5(8.9)$ & 23 & $5.1(16.5)$ & 24 & $3.4(18.4)$ & & & & & & \\
\hline Intervention & 37 & $33.9(9.9)$ & 33 & $-2.0(10.2)$ & 23 & $1.1(13.0)$ & 23 & $2.5(10.9)$ & $3.3(-1.0,7.5)$ & .13 & $-4.1(-11.8,3.6)$ & .29 & $-2.8(-10.0,4.4)$ & .44 \\
\hline \multicolumn{15}{|l|}{ POMA $(0-28)$} \\
\hline Usual care & 38 & $5.3(7.0)$ & 34 & $10.8(8.2)$ & & $-^{\dagger}$ & & $-^{\dagger}$ & & & & & & \\
\hline Intervention & 39 & $3.5(6.8)$ & 33 & $11.5(7.9)$ & & $-^{\dagger}$ & & $-{ }^{\dagger}$ & $-0.3(-6.5,5.8)$ & .90 & $-^{\dagger}$ & & $-^{\dagger}$ & \\
\hline \multicolumn{15}{|c|}{ Secondary outcomes } \\
\hline \multicolumn{15}{|c|}{ Activity restriction due to fear of falling (0-4) } \\
\hline Usual care & 38 & $0.7(1.0)$ & 34 & $-0.3(1.2)$ & 25 & $1.3(1.1)$ & 26 & $1.2(1.6)$ & & & & & & \\
\hline Intervention & 39 & $0.9(1.1)$ & 34 & $-0.2(1.1)$ & 23 & $1.2(1.4)$ & 24 & $0.7(1.4)$ & $0.1(-0.5,0.7)$ & .77 & $-0.1(0.8,0.7)$ & .82 & $-0.5(-1.2,0.3)$ & .23 \\
\hline \multicolumn{15}{|l|}{ FAC $(0-5)$} \\
\hline Usual care & 38 & $2.7(1.4)$ & 34 & $1.4(1.4)$ & 21 & $1.7(1.4)$ & 25 & $1.8(1.4)$ & & & & & & \\
\hline Intervention & 39 & $1.7(1.4)$ & 34 & $2.2(1.3)$ & 22 & $2.4(1.3)$ & 21 & $2.6(1.4)$ & $0.1(-0.3,0.5)$ & .62 & $0.0(-0.5,0.6)$ & .90 & $0.1(-0.6,0.8)$ & .75 \\
\hline
\end{tabular}

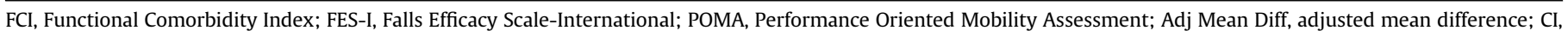
confidence interval.

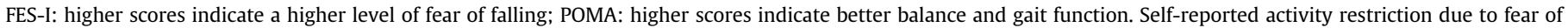

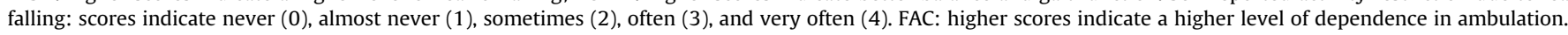
$\Delta$ indicates change score reflecting the difference between the score at any time point (Tx) and baseline score (T0).

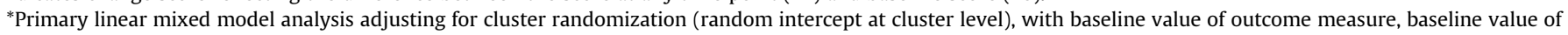

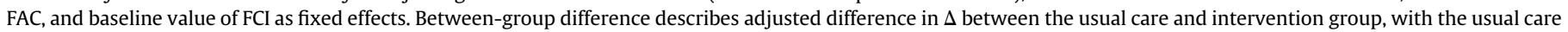
group as the reference category.

${ }^{\dagger}$ Not applicable, POMA was only assessed at discharge (T1), not at follow-up.

in patients with hip fracture. ${ }^{15}$ In recent literature, FoF has been approached and reconceptualized from perspectives from posttraumatic stress disorder (PTSD). ${ }^{39,40}$ In this context, Adamczewska and Nyman ${ }^{40}$ argue that the presence of anxiety is the key to whether FoF becomes maladaptive. In our study population, low scores were reported for symptoms of anxiety (median Hospital Anxiety and Depression Scale-subscale Anxiety scores at baseline 3.0 and 4.0 in the usual care group and the intervention group, respectively, with scores $>7$ indicating possible anxiety disorder). If anxiety has a critical role in the development of maladaptive or excessive forms of FoF, this would suggest an inappropriate selection of the target group in our study and may present a second reason for the absence of effect of the FIT-HIP intervention.

A possible explanation for the unexpected postintervention effect at discharge (ie, the reduction in FES-I score in the usual care group in contrast to the relatively consistent levels in the intervention group) is that treatment of FoF leads to increased awareness of fall risk and fallrelated concerns. This was also found by Faes and colleagues in the

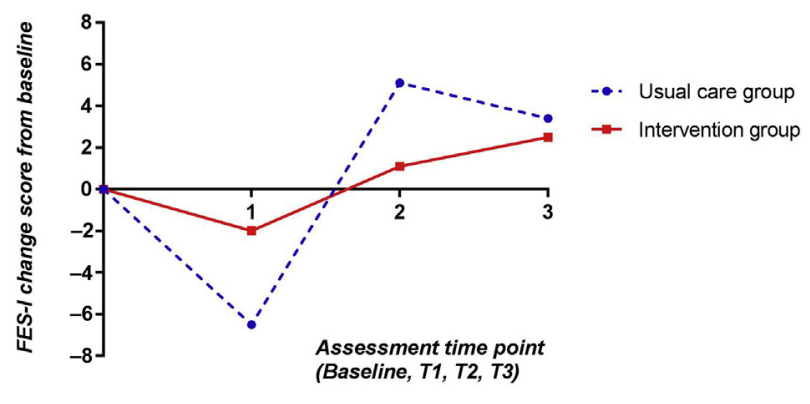

FES-I= Falls Efficacy Scale International. Range 16-64, higher score indicating a higher level of fear of falling. The Baseline assessment score was used as a reference for change score.

Fig. 2. FES-I change score at discharge and during follow-up. evaluation of a multifactorial falls prevention program aimed at reducing FoF in frail community-dwelling older adults. ${ }^{41}$ However, an important outcome in our study is that the differences in the level of FoF were not accompanied by differences in reported activity restriction due to FoF and physical performance (POMA/FAC). Furthermore, the effect identified in the usual care group was not sustained.

Considering that a different (ie, more advanced) timing may be more appropriate to target FoF, we can subsequently question whether the FIT-HIP intervention has the potential to effectively reduce FoF. To our knowledge, there are no comparable studies reporting on treatment of FoF in patients with hip fracture (although a protocol has been published $)^{42}$ or in other target groups in (geriatric) rehabilitation that are known to frequently report FoF. ${ }^{43}$ The cognitive behavioral approach used in the FIT-HIP intervention is based on intervention programs proven effective in reducing $\mathrm{FoF}$ in community-dwelling older adults. ${ }^{21-26}$ Various reviews have evaluated interventions for FoF in community-dwelling older people and found that efficacious interventions were typically multicomponent programs, combining exercise and cognitive behavioral therapy. ${ }^{19,20,44,45}$ Similarly, this was effective in reducing FoF in nursing home residents. ${ }^{46}$ In particular, cognitive restructuring, personal goal setting, promotion of physical activities, graded tasks, and behavioral practice are mentioned as core elements to reduce concerns about falls. $^{44,47}$ The key element of the FIT-HIP intervention is guided exposure to the feared activities, and this is embedded in physical therapy sessions, thereby representing the combination of a cognitive behavioral approach and exercise. Promotion of physical exercise is also an important part of our relapse prevention. Furthermore, cognitive restructuring is represented in the FIT-HIP intervention, although the intensity and duration may differ from other programs. Therefore, based on current knowledge and practice, the FIT-HIP intervention has (in theory) effective components to reduce FoF. The planned process evaluation of this trial will assess to what extent the intervention was performed according to the protocol. 
The results of this study demonstrate that management of FoF after recent hip fracture remains a challenge. In our opinion, several aspects of FoF after hip fracture need to be unraveled before proceeding to evaluate an intervention in a later phase of the rehabilitation process. We recommend that further research first focus on exploring the time-mediated effect of FoF after hip fracture, thereby gaining insight into how the direct physical consequences of hip fracture (eg, diminished muscle strength and balance, and dependence in ADL) influence and relate to FoF. Subsequently, it is important to evaluate how to distinguish the normal adaptive form of FoF from the dysfunctional and perhaps disproportionate form that requires treatment. A better understanding of the concept of FoF after fracture (including the possible mediating role of anxiety) can help in adequately assessing when, and to what extent, treatment for FoF is required.

To our knowledge, the FIT-HIP study is the first to evaluate and report on treatment for FoF after hip fracture. A major strength of the study is the cluster RCT design with a 6-month follow-up period. We could therefore limit contamination with regard to the complex intervention. ${ }^{48}$ As the transition to the home setting is probably a significant trigger for $\mathrm{FoF}^{43}$ it is important to evaluate long-term effects following discharge home, as we have done. A limitation of cluster randomization compared to individual randomization is the increased risk of imbalance in (observed and also unknown) baseline characteristics, as was seen in our data (FAC and FCI). However, sophisticated statistical techniques such as linear mixed models are able to take into account the clustering effects and adjust for the imbalance. Correcting for comorbidity did lead to a different outcome compared to less extensive models, which underpins the need to adjust for this established factor influencing outcome after hip fracture. $^{9,10,34}$ As the study was performed within inpatient multidisciplinary GR, the participants represent a particular group, and the results are not generalizable to all hip fracture patients. However, a general feature of the GR population (reflecting eligibility criteria for $\mathrm{GR}$ ) is that, based on the functional prognosis, there is reasonable probability that the patient will recover sufficiently to return home. This generally implies a reasonable prefracture functional ability and sufficient learning ability (no major cognitive deficits). This is in line with our inclusion criteria. However, there may be a underrepresentation of psychiatric symptoms in our study population (hence a relatively better functional prognosis). This may contribute to the ceiling effect seen for the FAC.

\section{Conclusion and Relevance}

This cluster RCT demonstrates that the FIT-HIP intervention was not effective in reducing FoF and improving mobility in geriatric rehabilitation after recent hip fracture. In order to adequately identify whether treatment of FoF is required, further research should explore the concept of FoF after fracture and differentiate between (1) FoF that can be considered a normal and adaptive response and (2) conditions when it is dysfunctional and disproportional.

For current clinical practice, we suggest to primarily focus on the FoF that hampers progress in functional recovery. We recommend routine screening of FoF at onset and evaluation of the rehabilitation treatment in order to observe the course of FoF and timely identify when FoF becomes maladaptive. Screening for comorbid anxiety also may be useful in this context. The existing treatment programs (eg, A Matter of Balance ${ }^{22-26}$ ) can be considered for treatment of maladaptive FoF in later stages of rehabilitation. When excessive or dysfunctional FoF is present in the initial phase of rehabilitation, we expect that a cognitive behavioral approach (such as guided exposure and cognitive restructuring) can be effective.

For an overview of the main insights and recommendations, see Appendix Table A4.

\section{Acknowledgments}

The authors express their gratitude to Bart Beck, health care psychologist, cognitive behavioral therapist and teacher at LUMC, for his assistance in developing the intervention, providing training to the intervention physiotherapists and psychologists, and his contribution to the interpretation of the findings. Thankfully, Eve Dumas and Eva van der Ploeg (both psychologists) were involved in designing the intervention and Frans van Wijngaarden (physiotherapist) was involved in training the research assistants in the physical assessments. We also thank our team of independent research assistants for their crucial role in enrollment/inclusion and data acquisition (Wilma van der Schrier, Olga Weeda, and Anita Pannekoek). In addition, we thank all participants and also the participating post-acute geriatric rehabilitation units and their staff for participating in the study, assisting with screening, and conducting the intervention. This study was performed within the University Network for the Care Sector South Holland in the Netherlands (UNC-ZH) (Reference: Achterberg WP, Caljouw MAA, Husebo BS. Towards academic nursing home medicine: A Dutch example for Norway? OMSORG 2015;1:10-15).

\section{References}

1. Wilson H. Orthogeriatrics in hip fracture. Open Orthop J 2017;11:1181-1189.

2. Giannoulis D, Calori GM, Giannoudis PV. Thirty-day mortality after hip fractures: Has anything changed? Eur J Orthop Surg Traumatol 2016;26: 365-370.

3. Asplin G, Carlsson G, Zidén L, et al. Early coordinated rehabilitation in acute phase after hip fracture-A model for increased patient participation. BMC Geriatr 2017:17:240.

4. Handoll HH, Cameron ID, Mak JC, et al. Multidisciplinary rehabilitation for older people with hip fractures. Cochrane Database Syst Rev; 2009:CD007125.

5. Crotty M, Unroe K, Cameron ID, et al. Rehabilitation interventions for improving physical and psychosocial functioning after hip fracture in older people. Cochrane Database Syst Rev; 2010:CD007624.

6. Nordström P, Thorngren KG, Hommel A, et al. Effects of geriatric team rehabilitation after hip fracture: Meta-analysis of randomized controlled trials. J Am Med Dir Assoc 2018;19:840-845.

7. Dyer SM, Crotty M, Fairhall N, et al. Fragility Fracture Network (FFN) Rehabilitation Research Special Interest Group. A critical review of the long-term disability outcomes following hip fracture. BMC Geriatr 2016;16:158.

8. Aarden JJ, van der Esch M, Engelbert RHH, et al. Hip fractures in older patients: Trajectories of disability after surgery. J Nutr Health Aging 2017;21:837-842.

9. Kristensen MT. Factors affecting functional prognosis of patients with hip fracture. Eur J Phys Rehabil Med 2011;47:257-264.

10. Morri M, Chiari P, Forni C, et al. What factors are associated with the recovery of autonomy after a hip fracture? A prospective, multicentric cohort study. Arch Phys Med Rehabil 2018;99:893-899.

11. Magaziner J, Simonsick EM, Kashner TM, et al. Predictors of functional recovery one year following hospital discharge for hip fracture: A prospective study. J Gerontol 1990;45:M101-M107.

12. Mossey JM, Mutran E, Knott K, et al. Determinants of recovery 12 months after hip fracture: The importance of psychosocial factors. Am J Public Health 1989; 79:279-286.

13. Oude Voshaar RC, Banerjee S, Horan M, et al. Fear of falling more important than pain and depression for functional recovery after surgery for hip fracture in older people. Psychol Med 2006;36:1635-1645.

14. Visschedijk J, Achterberg W, Van Balen R, et al. Fear of falling after hip fracture: A systematic review of measurement instruments, prevalence, interventions, and related factors. J Am Geriatr Soc 2015;1:10-15.

15. Visschedijk J, van Balen R, Hertogh C, et al. Fear of falling in patients with hip fractures: Prevalence and related psychological factors. J Am Med Dir Assoc 2013:14:218-220.

16. Bower ES, Wetherell JL, Petkus AJ, et al. Fear of falling after hip fracture: Prevalence, course, and relationship with one-year functional recovery. Am J Geriatr Psychiatry 2016;24:1228-1236.

17. Scheffer AC, Schuurmans MJ, van Dijk N, et al. Fear of falling: Measurement strategy, prevalence, risk factors and consequences among older persons. Age Ageing 2008:37:19-24.

18. Van der Meulen E, Zijlstra GA, Ambergen T, et al. Effect of fall-related concerns on physical, mental, and social function in community-dwelling older adults: A prospective cohort study. J Am Geriatr Soc 2014;62:2333-2338.

19. Zijlstra GA, van Haastregt JC, van Rossum E, et al. Interventions to reduce fear of falling in community-living older people: A systematic review. J Am Geriatr Soc 2007; 55:603-615.

20. Whipple MO, Hamel AV, Talley KMC. Fear of falling among community dwelling older adults: A scoping review to identify effective evidence-based interventions. Geriatr Nurs 2018;39:170-177. 
21. Tennstedt S, Howland J, Lachman M, et al. A randomized, controlled trial of a group intervention to reduce fear of falling and associated activity restriction in older adults. J Gerontol B Psychol Sci Soc Sci 1998;53:384-392.

22. Zijlstra GA, Tennstedt SL, van Haastregt JC, et al. Reducing fear of falling and avoidance of activity in elderly persons: The development of a Dutch version of an American intervention. Patient Educ Couns 2006;62:220-227.

23. Zijlstra GA, van Haastregt IC, Ambergen T, et al. Effects of a multicomponent cognitive behavioral group intervention on fear of falling and activity avoidance in community-dwelling older adults: Results of a randomized controlled trial. J Am Geriatr Soc 2009;57:2020-2028.

24. van Haastregt JC, Zijlstra GA, Hendriks MR, et al. Cost-effectiveness of an intervention to reduce fear of falling. Int J Technol Assess Health Care 2013;29: 219-226.

25. Zijlstra GA, van Haastregt JC, Du Moulin MF, et al. Effects of the implementation of an evidence-based program to manage concerns about falls in older adults. Gerontologist 2013;53:839-849.

26. Dorresteijn TA, Zijlstra GA, Ambergen AW, et al. Effectiveness of a home-based cognitive behavioral program to manage concerns about falls in communitydwelling, frail older people: Results of a randomized controlled trial. BMC Geriatr 2016;16:2.

27. Holstege MS, Caljouw MAA, Zekveld IG, et al. Successful geriatric rehabilitation: Effects on patients' outcome of a national program to improve quality of care, the SINGER Study. J Am Med Dir Assoc 2017;18:383-387.

28. Scheffers-Barnhoorn MN, van Haastregt JC, Schols JM, et al. A multi-component cognitive behavioural intervention for the treatment of fear of falling after hip fracture (FIT-HIP): protocol of a randomised controlled trial. BMC Geriatr 2017;17:71.

29. Meijer R, van Limbeek J, de Haan R. Development of the stroke-unit discharge guideline: Choice of assessment instruments for prediction in the subacute phase post-stroke. Int J Rehabil Res 2006;29:1-8.

30. Gillespie LD, Clare Robertson M, Gillespie WJ, et al. Interventions for preventing falls in older people living in the community. Cochrane Database Syst Rev; 2012:CD007146.

31. Verenso. Elderly care physicians in the Netherlands: Professional profile and competencies. 2015. Available at: https://www.verenso.nl/_asset/_public/ Vereniging/VER0026_Professionalprofile_broch_DEF.pdf. Accessed April 12, 2019.

32. Visschedijk JH, Terwee CB, Caljouw MA, et al. Reliability and validity of the Falls Efficacy Scale-International after hip fracture in patients aged $\geq 65$ years. Disabil Rehabil 2015;37:2225-2232.

33. Faber MJ, Bosscher RJ, van Wieringen PC. Clinimetric properties of the performance-oriented mobility assessment. Phys Ther 2006;86:944-954.

34. Lim KK, Yeo W, Koh JSB, et al. The role of prefracture health status in physical and mental function after hip fracture surgery. J Am Med Dir Assoc 2018;19: 989-994.
35. McKee KJ, Orbell S, Austin CA, et al. Fear of falling, falls efficacy, and health outcomes in older people following hip fracture. Disabil Rehabil 2002;24: 327-333.

36. Portegijs E, Edgren J, Salpakoski A et al. Balance confidence was associated with mobility and balance performance in older people with fall-related hip fracture: A cross-sectional study. Arch Phys Med Rehabil 2012;93: 2340-2346.

37. Jellesmark A, Herling SF, Egerod I, et al. Fear of falling and changed functional ability following hip fracture among community-dwelling elderly people: An explanatory sequential mixed method study. Disabil Rehabil 2012;34: 2124-2131.

38. Payette MC, Bélanger C, Léveillé V, et al. Fall-related psychological concerns and anxiety among community-dwelling older adults: Systematic review and meta-analysis. PLoS One 2016;11:e0152848.

39. Bloch F. A new accurate clinical assessment tool for fear of falling is needed for older patients. J Am Med Dir Assoc 2019;20:220-221.

40. Adamczewska N, Nyman SR. A new approach to fear of falls from connections with the posttraumatic stress disorder literature. Gerontol Geriatr Med 2018;4: $1-7$.

41. Faes MC, Reelick MF, Melis RJ, et al. Multifactorial fall prevention for pairs of frail community-dwelling older fallers and their informal caregivers: A dead end for complex interventions in the frailest fallers. J Am Med Dir Assoc 2011; $12: 451-458$.

42. Kampe K, Kohler M, Albrecht D, et al. Hip and pelvic fracture patients with fear of falling: Development and description of the "Step by Step" treatment protocol. Clin Rehabil 2017;31:571-581.

43. Visschedijk JH, Caljouw MA, Bakkers E, et al. Longitudinal follow-up study on fear of falling during and after rehabilitation in skilled nursing facilities. BMC Geriatr 2015;15:161.

44. Liu TW, Ng GYF, Chung RCK, et al. Cognitive behavioural therapy for fear of falling and balance among older people: A systematic review and meta-analysis. Age Ageing 2018;47:520-527.

45. Kendrick D, Kumar A, Carpenter H, et al. Exercise for reducing fear of falling in older people living in the community. Cochrane Database Syst Rev; 2014:CD009848.

46. Huang TT, Chung ML, Chen FR, et al. Evaluation of a combined cognitivebehavioural and exercise intervention to manage fear of falling among elderly residents in nursing homes. Aging Ment Health 2016;20:2-12.

47. Vestjens L, Kempen GI, Crutzen R, et al. Promising behavior change techniques in a multicomponent intervention to reduce concerns about falls in old age: A Delphi study. Health Educ Res 2015;30:309-322.

48. Flicker L. Intervention research in nursing homes: The rise and rise of the cluster randomized trial. J Am Med Dir Assoc 2008;9:213-214. 
Appendix

Table A1

Linear Mixed Model Structure Used in the FIT-HIP Study

\begin{tabular}{|c|c|}
\hline Primary Model & \\
\hline \multirow[t]{4}{*}{ Fixed effects (all outcome measures) } & Treatment group* \\
\hline & Baseline value outcome measure \\
\hline & Baseline value FAC \\
\hline & Baseline value FCI \\
\hline \multirow[t]{2}{*}{ Additional fixed effects (for outcome measures with assessments at $\mathrm{T} 2$ and $\mathrm{T} 3)^{\dagger}$} & Time (T1-T3) \\
\hline & Interaction term: treatment group $\times$ time \\
\hline Random effects & Intercept $^{\ddagger}$ \\
\hline Covariance structure & Factor-analytic first order \\
\hline
\end{tabular}

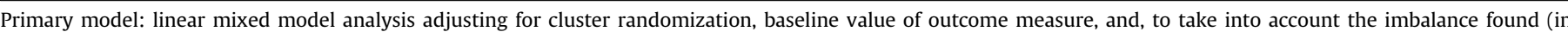

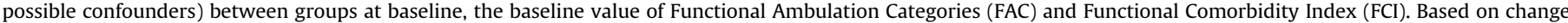

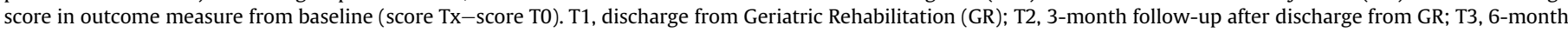
follow-up after discharge from GR.

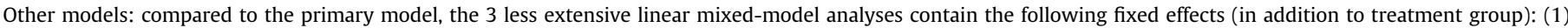
baseline value outcome measure; (2) baseline value outcome measure + FAC score; (3) baseline value outcome measure + baseline FCI score.

*Treatment group: [usual care group] or [intervention group].

†Outcome measures with assessments at T2 and T3: Falls Efficacy Scale-International (FES-I); FAC; and self-reported activity restriction due to fear of falling.

${ }^{\ddagger}$ Random intercept accounting for dependence within a cluster.

${ }^{\S}$ Based on Akaike's information criterion. Factor-analytic first order is a covariance structure that allows temporal correlation (ie, accounts for dependence between multiple measurements of the same patient).

Table A2

Treatment Effects of FIT-HIP Intervention-All Linear Mixed Models

\begin{tabular}{|c|c|c|c|c|c|c|}
\hline & \multicolumn{6}{|c|}{ Between-Group Differences: Intervention vs Usual Care Group* } \\
\hline & \multicolumn{2}{|l|}{$\mathrm{T} 1$} & \multicolumn{2}{|l|}{$\mathrm{T} 2$} & \multicolumn{2}{|l|}{ T3 } \\
\hline & Adj Mean Diff (95\% CI) & $P$ Value & Adj Mean Diff (95\% CI) & $P$ Value & Adj Mean Diff (95\% CI) & $P$ Value \\
\hline \multicolumn{7}{|l|}{ FES-I (16-64) } \\
\hline Adjusted for: baseline FES-I & $4.9(1.1,8.7)$ & .013 & $-2.6(-10.1,4.9)$ & .50 & $-0.7(-7.6,6.3)$ & .85 \\
\hline Adjusted for: baseline FES-I + FAC & $4.4(0.4,8.5)$ & .033 & $-3.0(-10.5,4.5)$ & .43 & $-1.1(-8.1,5.9)$ & .75 \\
\hline Adjusted for: baseline FES-I + FCI & $3.5(-0.5,7.5)$ & .09 & $-3.9(-11.6,3.7)$ & .31 & $-2.6(-9.7,4.5)$ & .47 \\
\hline Adjusted for: baseline FES-I + FAC + FCI & $3.3(-1.0,7.5)$ & .13 & $-4.1(-11.8,3.6)$ & .29 & $-2.8(-10.0,4.4)$ & .44 \\
\hline \multicolumn{7}{|l|}{ POMA $(0-28)$} \\
\hline Adjusted for: baseline POMA & $-0.7(-3.8,2.4)$ & 64 & $-^{\dagger}$ & & $-^{\dagger}$ & \\
\hline Adjusted for: baseline POMA + FAC & $-0.2(-3.5,3.2)$ & .92 & $-^{\dagger}$ & & $-一^{\dagger}$ & \\
\hline Adjusted for: baseline POMA + FCI & $-0.3(-6.4,5.7)$ & .89 & $-^{\dagger}$ & & $-^{\dagger}$ & \\
\hline Adjusted for: baseline POMA + FAC + FCI & $-0.3(-6.5,5.8)$ & .90 & $-^{\dagger}$ & & $-^{\dagger}$ & \\
\hline \multicolumn{7}{|c|}{ Activity restriction due to fear of falling (AR) (0-4) } \\
\hline Adjusted for: baseline AR & $0.2(-0.3,0.8)$ & .41 & $0.0(-0.7,0.7)$ & .96 & $-0.4(-1.1,0.4)$ & .33 \\
\hline Adjusted for: baseline AR + FAC & $0.2(-0.4,0.7)$ & .50 & $0.0(-0.7,0.7)$ & .90 & $-0.4(-1.1,0.4)$ & .30 \\
\hline Adjusted for: baseline AR + FCI & $0.1(-0.5,0.7)$ & 68 & $-0.1(-0.8,0.7)$ & .87 & $-0.4(-1.2,0.3)$ & .24 \\
\hline Adjusted for: baseline AR + FAC + FCI & $0.1(-0.5,0.7)$ & .77 & $-0.1(-0.8,0.7)$ & .82 & $-0.5(-1.2,0.3)$ & .23 \\
\hline \multicolumn{7}{|l|}{ FAC $(0-5)$} \\
\hline Adjusted for: baseline FAC & $0.0(-0.4,0.4)$ & .92 & $-0.1(-0.6,0.5)$ & .85 & $0.0(-0.6,0.6)$ & 1.00 \\
\hline Adjusted for: baseline FAC + FCI & $0.1(-0.3,0.5)$ & 62 & $0.0(-0.5,0.6)$ & .90 & $0.1(-0.6,0.8)$ & .75 \\
\hline
\end{tabular}

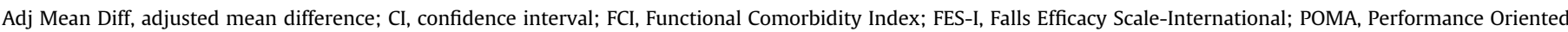

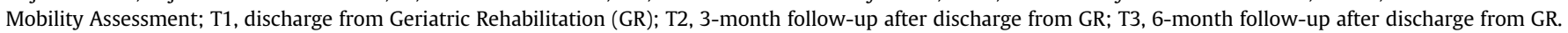

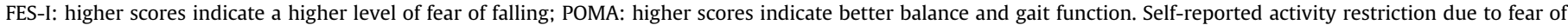
falling: scores indicate never (0), almost never (1), sometimes (2), often (3) and very often (4); FAC: higher scores indicate a higher level of dependence in ambulation.

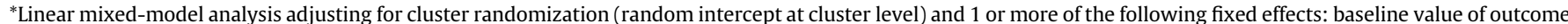

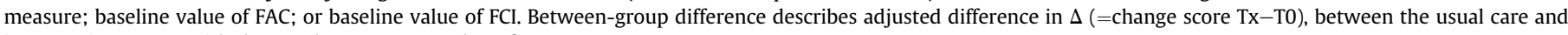
intervention group, with the usual care group as the reference category.

†Not applicable, POMA was only assessed at discharge (T1), not at follow-up. 
Table A3

Adverse Events Reported During the FIT-HIP Study

\begin{tabular}{|c|c|c|c|c|}
\hline & \multicolumn{2}{|c|}{ Usual Care Group } & \multicolumn{2}{|c|}{ Intervention Group } \\
\hline & $\begin{array}{l}\text { During } \\
\text { GR }\end{array}$ & Follow-up & $\begin{array}{l}\text { During } \\
\text { GR }\end{array}$ & Follow-up \\
\hline Death, n & 2 & 0 & 0 & 3 \\
\hline \multicolumn{5}{|l|}{ Hospital readmissions, $\mathrm{n}$} \\
\hline Total amount of readmissions & 3 & 5 & 3 & 2 \\
\hline Participants with readmission(s) & 3 & 4 & 2 & 2 \\
\hline \multicolumn{5}{|l|}{ Fall events, $\mathrm{n}$} \\
\hline Total amount of fall events & 12 & 19 & 4 & 5 \\
\hline Participants with fall event(s) & 4 & 10 & 4 & 4 \\
\hline Fracture(s) due to fall event* & 0 & 1 & 0 & 2 \\
\hline
\end{tabular}

GR, inpatient multidisciplinary Geriatric Rehabilitation.

Follow-up included assessments at 3 and 6 months after discharge from Geriatric Rehabilitation.

*Usual care group: fracture of the thumb; intervention group: ankle fracture and second hip fracture.

Table A4

Main Insights From the FIT-HIP Study

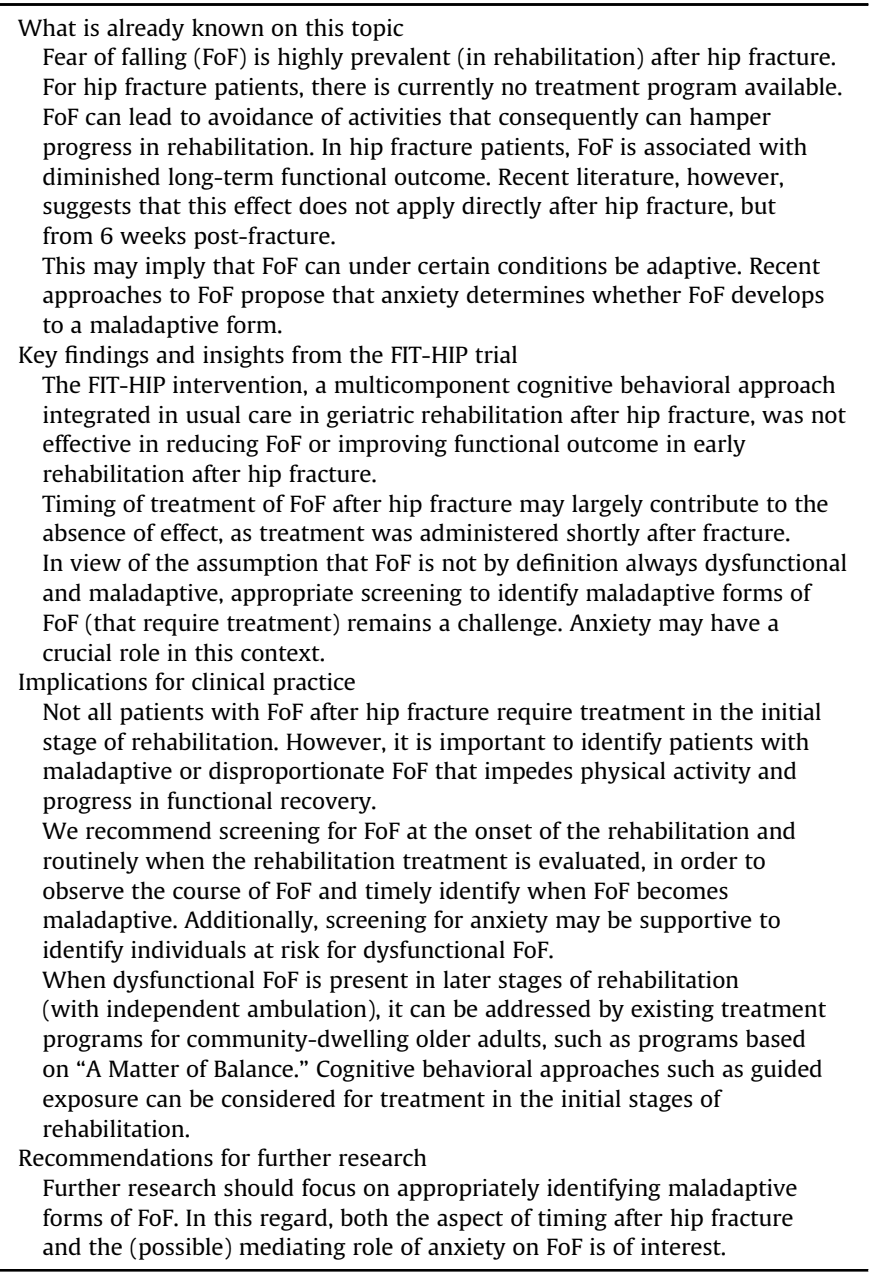

